A. Koriyama

Nagoya Math. J.

Vol. 55 (1974), 91-110

\title{
ON LIE ALGEBRAS OF VECTOR FIELDS WITH INVARIANT SUBMANIFOLDS
}

\author{
AKIRA KORIYAMA
}

\section{§ 0. Introduction.}

It is known (Pursell and Shanks [9]) that an isomorphism between Lie algebras of infinitesimal automorphisms of $C^{\infty}$ structures with compact support on manifolds $M$ and $M^{\prime}$ yields an isomorphism between $C^{\infty}$ structures of $M$ and $M^{\prime}$.

Omori [5] proved that this is still true for some other structures on manifolds. More precisely, let $M$ and $M^{\prime}$ be Hausdorff and finite dimensional manifolds without boundary. Let $\alpha$ be one of the following structures :

(1) $C^{\infty}$-structures, $(\alpha=\phi)$

(2) $S L$-structure, i.e. a volume element (positive $n$-form) with a non-zero constant multiplicative factor, $(\alpha=d V)$

(3) $S p$-(symplectic) structure, i.e. symplectic 2-form with a non-zero constant multiplicative factor, $(\alpha=\Omega)$

(4) Contact structure, i.e. contact 1 -form with a non-zero $C^{\infty}$-function as a multiplicative factor, $(\alpha=\omega)$

(5) Fibring with compact fibre, $(\alpha=\mathscr{F})$

Let $\alpha$ (resp. $\alpha^{\prime}$ ) be one of the above structures on $M$ (resp. $M^{\prime}$ ). Let $\Gamma_{\alpha}\left(T_{M}\right)$ be the Lie algebra of all $C^{\infty}, \alpha$-preserving infinitesimal transformations with compact support. We denote by $\mathscr{D}_{\alpha}(M)$ the group of all $C^{\infty}, \alpha$-preserving diffeomorphisms on $M$ with compact support, that is, identity outside a compact subset. Then we have the following theorem

THEOREM (Omori [5]). $\Gamma_{\alpha}\left(T_{M}\right)$ is algebraically isomorphic to $\Gamma_{\alpha^{\prime}}\left(T_{M^{\prime}}\right)$, if and only if $(M, \alpha)$ is isomorphic to $\left(M^{\prime}, \alpha^{\prime}\right)$. Especially, $\mathscr{D}_{\alpha}(M)$ is isomorphic to $\mathscr{D}_{\alpha^{\prime}}\left(M^{\prime}\right)$.

Received December 17, 1973.

Revised May 9, 1974. 
Omori $[6,7,8]$ defined the notion of I.L.H.-Lie group and proved that the group $\mathscr{D}_{\alpha}(M)$ stated above is an I.L.H.-Lie group. As a matter of fact, $\mathscr{D}_{\alpha}(M)$ is a (strong) I.L.H.-Lie group with the Lie algebra $\Gamma_{\alpha}\left(T_{M}\right)$. So we can say that the I.L.H.-Lie group $\mathscr{D}_{\alpha}(M)$ is determined by its Lie algebra.

Let $(M, N)$ be a pair of paracompact $C^{\infty}$ manifolds such that $N$ is a closed submanifold of $M$ (may be $\operatorname{dim} N=0$ ). We denote by $\Gamma_{N}\left(T_{M}\right)$ the Lie algebra of all $C^{\infty}, N$-preserving, i.e. tangent to $N$, infinitesimal transformations with compact support. By $\mathscr{D}(M, N)$ we denote the group of all $C^{\infty}, N$-preserving diffeomorphisms on $M$ with compact support. The purpose of this paper is to prove the following theorem.

THEOREM. $\quad \Gamma_{N}\left(T_{M}\right)$ is algebraically isomorphic to $\Gamma_{N^{\prime}}\left(T_{M^{\prime}}\right)$, if and only if there exists a $C^{\infty}$ diffeomorphism $\varphi: M \rightarrow M^{\prime}$ such that $\varphi(N)=N^{\prime}$. Especially $\mathscr{D}(M, N)$ is isomorphic to $\mathscr{D}\left(M^{\prime}, N^{\prime}\right)$.

If $M$ is compact, then $\mathscr{D}(M, N)$ becomes an I.L.H.-Lie subgroup of $\mathscr{D}(M)$ with the Lie algebra $\Gamma_{N}\left(T_{M}\right)$ (Ebin and Marsden [2]). So in this case we can say that $\mathscr{D}(M, N)$ is determined as an I.L.H.-Lie group by its Lie algebra.

The proof of our theorem is parallel to that of Pursell and Shanks. Main parts of our proof are $\S 2$ and $\S 3$. We denote by $\Gamma_{0}\left(T_{M}\right)$ instead of $\Gamma_{N}\left(T_{M}\right)$ for the case $N=\left\{p_{0}\right\}$, where $p_{0} \in M$ is an arbitrary but fixed point. Since the structure of $\Gamma_{0}\left(T_{M}\right)$ is different from that of $\Gamma_{N}\left(T_{M}\right)$ for $\operatorname{dim} N \geqq 1$, we will investigate $\Gamma_{0}\left(T_{M}\right)$ and $\Gamma_{N}\left(T_{M}\right)$ separately, that is, in $\S 2$ we will study maximal ideals of $\Gamma_{0}\left(T_{M}\right)$ and in $\S 3$ that of $\Gamma_{N}\left(T_{M}\right)$.

The author would like to express his gratitude to Prof. H. Omori for suggesting the problem and also for his helpfull advice.

\section{§ 1. Preliminaries.}

Let $\boldsymbol{R}^{n} \times \boldsymbol{R}^{\ell}$ be the euclidean space with coordinates $\left\{x^{1}, \cdots, x^{n}, y^{1}\right.$, $\left.\cdots, y^{\ell}\right\}$. Let $\mathscr{F}=C^{\infty}\left(\boldsymbol{R}^{n} \times \boldsymbol{R}^{\ell}\right)$ be the set of all $C^{\infty}$ functions on $\boldsymbol{R}^{n} \times \boldsymbol{R}^{\ell}$. Let $\mathscr{G}=C^{\infty}\left(\boldsymbol{R}^{n} \times 0\right)=C^{\infty}\left(\boldsymbol{R}^{n}\right)$ be the set of all $C^{\infty}$ functions on $\boldsymbol{R}^{n}$. $\mathscr{G}$ is naturally identified with the subset of $C^{\infty}\left(\boldsymbol{R}^{n} \times \boldsymbol{R}^{\ell}\right)$ by the projection $\boldsymbol{R}^{n} \times \boldsymbol{R}^{\ell} \rightarrow \boldsymbol{R}^{n}$. Let $\mathscr{I}$ be the ideal of $\mathscr{F}$ of functions vanishing on $\boldsymbol{R}^{n} \times 0$, i.e.

$$
\mathscr{I}=\left\{f \in \mathscr{F}|f|_{\boldsymbol{R}^{n \times 0}}=0\right\}
$$


Clearly $x^{i} \in \mathscr{G}(i=1, \cdots, n)$ and $y^{\alpha} \in \mathscr{I}(\alpha=1, \cdots, \ell)$.

LEMMA 1.1. For any $f \in \mathscr{F}$ there exist $g_{0} \in \mathscr{G}$ and $f_{\alpha} \in \mathscr{F}(1 \leqq \alpha \leqq \ell)$ such that $f=g_{0}+y^{1} f_{1}+\cdots+y^{\ell} f_{\ell}$.

Proof. Easy computation. (see, for example, [1])

CoRollary 1.2. If $f \in \mathscr{I}$, then $g_{0}=0$.

Let $M$ be a $C^{\infty}$ manifold of dimension $m$, and $N$ be a closed submanifold of dimension $n$ such that $n \geqq 0$. We set $\ell=m-n$.

LEMMA 1.3. The subset $\Gamma_{N}\left(T_{M}\right)$ of $\Gamma\left(T_{M}\right)$ is a Lie subalgebra of $\Gamma\left(T_{M}\right)$.

Proof. Let $\left(U ; x^{1}, \cdots, x^{n}, y^{1}, \cdots, y^{\ell}\right)$ be a coordinate system at $p \in N$ such that $U \cap N=\left\{y^{1}=\cdots=y^{\ell}=0\right\}$. Let $X=\xi^{i}\left(\partial / \partial x^{i}\right)+\xi^{\alpha}\left(\partial / \partial y^{\alpha}\right)$ and $Y=\eta^{i}\left(\partial / \partial x^{i}\right)+\eta^{\alpha}\left(\partial / \partial y^{\alpha}\right)$ be in $\Gamma_{N}\left(T_{M}\right)$. Then by Corollary $1.2 \xi^{\alpha}$ and $\eta^{\alpha}$ are written as

$$
\xi^{\alpha}=y^{1} \xi_{1}^{\alpha}+\cdots+y^{\ell} \xi_{\ell}^{\alpha} \quad \text { and } \eta^{\alpha}=y^{1} \eta_{1}^{\alpha}+\cdots+y^{\ell} \eta_{\ell}^{\alpha} \quad(\alpha=1, \cdots, \ell),
$$

where $\xi_{s}^{\alpha}, \eta_{s}^{\alpha} \in C^{\infty}(M)(s=1, \cdots, \ell)$. We have then

$$
[X, Y]=\left(\xi^{i} \frac{\partial \eta^{j}}{\partial x^{i}}-\eta^{i} \frac{\partial \xi^{j}}{\partial x^{i}}\right) \frac{\partial}{\partial x^{j}} \quad \text { on } U \cap N .
$$

Hence $[X, Y] \in \Gamma_{N}\left(T_{M}\right)$.

Lemma 1.4. For each $X \in \Gamma_{N}\left(T_{M}\right) r_{*}(X)$ denotes the restriction of $X$ to $N$. Then $r_{*}$ is a Lie algebra homomorphism of $\Gamma_{N}\left(T_{M}\right)$ onto $\Gamma\left(T_{N}\right)$, that is, $r_{*}[X, Y]=\left[r_{*} X, r_{*} Y\right]$.

Proof. Easy computation.

LemMa 1.5. Let $X \in \Gamma_{N}\left(T_{M}\right)$ such that $X(p) \neq 0$ at $p \in M$. Then there is a local coordinate system $\left(U ; x^{1}, \cdots, x^{n}, y^{1}, \cdots, y^{\ell}\right)$ such that $X=$ $\partial / \partial x^{1}$ on $U$ and if $p \in N$ and $\operatorname{dim} N \geqq 1$ then $U \cap N=\left\{y^{1}=\cdots=y^{\ell}=0\right\}$.

Proof. Easy computation.

\section{§ 2. Characterization of maximal ideals of $\Gamma_{0}\left(T_{M}\right)$.}

We denote by $\Gamma\left(T_{M}\right)$ the Lie algebra of all $C^{\infty}$ vector fields on $M$ with compact support, and $\Gamma_{0}\left(T_{M}\right)=\left\{X \in \Gamma\left(T_{M}\right) \mid X\left(p_{0}\right)=0\right\}$ is a Lie subalgebra of $\Gamma\left(T_{M}\right)$, where $p_{0} \in M$ is an arbitrary but fixed point. We set 


$$
\Gamma_{0}^{k}\left(T_{M}\right)=\left\{X \in \Gamma_{0}\left(T_{M}\right) \mid \dot{\mathfrak{l}}^{r}(X)\left(p_{0}\right)=0 \text { for all } r \leqq k\right\},
$$

where $j^{r}(X)\left(p_{0}\right)$ is the $r$-jet of $X$ at $p_{0}$.

LEMMA 2.1. If $X \in \Gamma_{0}\left(T_{M}\right)$ does not vanish at $p \in M\left(p \neq p_{0}\right)$, then for any $Z \in \Gamma_{0}\left(T_{M}\right)$ there are a neighborhood $U$ of $p$ in $M$ and a vector field $Y \in \Gamma_{0}\left(T_{M}\right)$ such that $[X, Y]=Z$ on $U$.

Proof. By Lemma 1.5 there exists a local coordinate system ( $V$; $\left.x^{1}, \cdots, x^{m}\right)$ at $p$ such that $X=\partial / \partial x^{1}$ on $V$. For any $Z=\zeta^{i}\left(\partial / \partial x^{i}\right) \in \Gamma_{0}\left(T_{M}\right)$, i.e. $Z \in \Gamma_{0}\left(T_{M}\right)$ such that $\left.Z\right|_{V}=\zeta^{i}\left(\partial / \partial x^{i}\right)$, we consider the differential equations

$$
\frac{\partial \eta^{i}}{\partial x^{1}}=\zeta^{i} \quad(i=1, \cdots, m)
$$

These equations have solutions on some neighborhood $U \subset V$ of $p$. Set $Y=\eta^{i}\left(\partial / \partial x^{i}\right)$, then $Y$ is a $C^{\infty}$ vector field on $U$ and satisfies the equation $[X, Y]=Z$ on $U$. Here we may assume that $U$ is relatively compact in $V$ and dose not contain $p_{0}$. Then an appropriate extension of $Y$ is contained in $\Gamma_{0}\left(T_{M}\right)$.

LEMMA 2.2. Let $\mathrm{gl}(m)$ be the Lie algebra of all $m \times m$ real matrices. Then we have the following results.

(i) $\mathrm{s} \ell(m)=\{A \in \mathfrak{g l}(m) \mid \operatorname{trace} A=0\}$ is an ideal of $\operatorname{gl}(m)$.

(ii) The center of $\operatorname{gr}(m)$ is $z=\{\lambda I \mid I$ is the unit matrix and $\lambda$ is a real number.\}, and $z$ is an ideal of $\mathfrak{g l}(m)$.

(iii) If $m \geqq 2$, then $\operatorname{gl}(m)=z \oplus s \ell(m)$ (direct sum), i.e. $z \cap s \ell(m)=0$. If $m=1$, then $\mathfrak{g l}(m)=z$.

(iv) If $m \geqq 2$, then $s \ell(m)$ is a simple Lie algebra, that is, $s \ell(m)$ does not admit any non-trivial ideals.

(v) z and $s \ell(m)$ are maximal ideals of $\mathfrak{g l}(m)$.

Proof. These results are well known, and we omit the proofs. (see, for example, [3])

LEMMA 2.3. For each point $p \in M$ such that $p \neq p_{0}$ we denote by $\mathscr{I}_{p}$ the subset $\left\{X \in \Gamma_{0}\left(T_{M}\right) \mid X(p)=0\right.$ and $\dot{i}^{r}(X)(p)=0$ for all $\left.r \geqq 1\right\}$ of $\Gamma_{0}\left(T_{M}\right)$. Then for each $p \in M, \mathscr{I}_{p}$ is an ideal of $\boldsymbol{\Gamma}_{0}\left(T_{M}\right)$.

Proof. The proof is direct computation. 
LEMMA 2.4. Let $p \in M$ be a given point such that $p \neq p_{0}$. If $\mathscr{I}$ is a proper ideal of $\Gamma_{0}\left(T_{M}\right)$, i.e. $\mathscr{I} \subsetneq \Gamma_{0}\left(T_{M}\right)$, such that $X(p)=0$ for all $X \in \mathscr{I}$. Then $\mathscr{I} \subset \mathscr{I}_{p}$.

Proof. Since $p \neq p_{0}$, there is a local coordinate system $\left(U ; x^{1}, \cdots\right.$, $\left.x^{m}\right)$ at $p$ such that $\bar{U} \nexists p_{0}$. Hence appropriate extensions of $\partial / \partial x^{j}(j=1$, $\cdots, m)$ are contained in $\Gamma_{0}\left(T_{M}\right)$. We also denote the extended vector fields by the same letters. For any $X=\xi^{i}\left(\partial / \partial x^{i}\right) \in \mathscr{I}$ we have $\left[\partial / \partial x^{j}, X\right]$ $=\partial \xi^{i} / \partial x^{j} \cdot \partial / \partial x^{i}$ for all $j=1, \cdots, m$. Since $\mathscr{I}$ is an ideal, $\left[\partial / \partial x^{j}, X\right] \in \mathscr{I}$. From the assumption for $\mathscr{I},\left(\partial \xi^{i} / \partial x^{j}\right)(p)=0$ for all $i, j=1, \cdots, m$. By induction on $r$, we have $\dot{j}^{r}(X)(p)=0$ for all $r \geqq 1$. Therefore $\mathscr{I} \subset \mathscr{I}_{p}$.

LEMMA 2.5. Let $A$ be an arbitrary Lie algebra. If $\mathfrak{a}$ and $\mathfrak{b}$ are ideals of $A$ such that $\mathfrak{a} \supset \mathfrak{b}$. Then $(A / \mathfrak{b}) /(\mathfrak{a} / \mathfrak{b}) \cong A / \mathfrak{a}$.

Proof. The result is well known, and we omit the proof.

LEMMA 2.6. The subset $\Gamma_{0}^{1}\left(T_{M}\right)=\left\{X \in \Gamma_{0}\left(T_{M}\right) \mid \dot{\mathfrak{j}}^{1}(X)\left(p_{0}\right)=0\right\}$ is a proper ideal of $\Gamma_{0}\left(T_{M}\right)$.

Proof. Easy computation.

LEMMA 2.7. Let $\pi: \Gamma_{0}\left(T_{M}\right) \rightarrow \Gamma_{0}\left(T_{M}\right) / \Gamma_{0}^{1}\left(T_{M}\right) \cong \mathfrak{g l}(m)$ be the natural projection. We define $\mathscr{I}_{z}$ and $\mathscr{I}_{s \ell}$ by $\mathscr{I}_{z}=\pi^{-1}\left(z^{\prime}\right)$ and $\mathscr{I}_{s \ell}=\pi^{-1}(s \ell(m))$. Then both $\mathscr{I}_{z}$ and $\mathscr{I}_{\text {se }}$ are proper ideals of $\Gamma_{0}\left(T_{M}\right)$.

Proof. Since $\pi: \Gamma_{0}\left(T_{M}\right) \rightarrow \mathfrak{g l}(m)$ is an onto Lie algebra homomorphism, we have the desired result.

Proposition 2.8. If $\mathfrak{m}$ is a maximal of ideal $\boldsymbol{\Gamma}_{0}\left(T_{M}\right)$ such that $\mathfrak{m} \supset$ $\Gamma_{0}^{1}\left(T_{M}\right)$, then $\mathfrak{m}=\mathscr{I}_{z}$ or $\mathscr{I}_{s \ell}$.

Proof. Let $\mathfrak{m} \subsetneq \Gamma_{0}\left(T_{M}\right)$ be a maximal ideal such that $\mathfrak{m} \supset \Gamma_{0}^{1}\left(T_{M}\right)$. Then by Lemma $2.5 \mathrm{~m} / \Gamma_{0}^{1}\left(T_{M}\right)$ is a proper ideal of $\Gamma_{0}\left(T_{M}\right) / \Gamma_{0}^{1}\left(T_{M}\right)$. By Lemma 2.2, $\boldsymbol{\Gamma}_{0}\left(T_{M}\right) / \Gamma_{0}^{1}\left(T_{M}\right) \cong \mathfrak{g l}(m)=z \oplus s \ell(m)$ and both $z$ and $s \ell(m)$ are simple Lie algebras. Hence $m / \Gamma_{0}^{1}\left(T_{M}\right)$ should be equal to either $z$ or $s \ell(m)$. Therefore we have $\mathfrak{m}=\pi^{-1}(\mathfrak{z})=\mathscr{I}_{z}$ or $\mathfrak{m}=\pi^{-1}(s \ell(m))=\mathscr{I}_{s \ell}$.

LEMMA 2.9. If $\mathfrak{m}$ is a maximal ideal of $\Gamma_{0}\left(T_{M}\right)$ such that $\mathfrak{m} \supset \Gamma_{0}^{\infty}\left(T_{M}\right)$, then for any point $p \neq p_{0}$, there exists an element $X \in \mathfrak{M}$ such that $X(p)$ $\neq 0$, where

$$
\Gamma_{0}^{\infty}\left(T_{M}\right)=\left\{X \in \Gamma_{0}\left(T_{M}\right) \mid \dot{j}^{r}(X)\left(p_{0}\right)=0 \text { for all } r \geqq 1\right\} .
$$


Proof. Assume that there exists a point $p \in M\left(p \neq p_{0}\right)$ such that $X(p)=0$ for all $X \in \mathfrak{m}$. By Lemma $2.4 \mathfrak{m} \subset \mathscr{I}_{p}$. Since $\mathfrak{m}$ is a maximal ideal, $\mathfrak{m}=\mathscr{I}_{p}$. On the other hand, since $p \neq p_{0}$, there exists $Y \in \Gamma_{0}^{\infty}\left(T_{M}\right)$ such that $Y(p) \neq 0$. Hence $\mathfrak{m}=\mathscr{I}_{p} \nexists Y$, contradicting the condition $\mathfrak{m} \supset \Gamma_{0}^{\infty}\left(T_{M}\right)$.

LEMMA 2.10. If $\mathfrak{m}$ is a maximal ideal of $\Gamma_{0}\left(T_{M}\right)$ such that $\mathfrak{m} \supset \Gamma_{0}^{\infty}\left(T_{M}\right)$, then $\mathfrak{j}^{1}(\mathfrak{m})\left(p_{0}\right)$ is a proper ideal of $\mathrm{gl}(m)$, where $\mathfrak{i}^{1}(\mathfrak{m})\left(p_{0}\right)$ is the image of $\mathfrak{m}$ under the natural projection

$$
\pi: \Gamma_{0}\left(T_{M}\right) \rightarrow \Gamma_{0}\left(T_{M}\right) / \Gamma_{0}^{1}\left(T_{M}\right) \cong \mathfrak{g l}(m) .
$$

Proof. Assume $\mathfrak{j}^{1}(\mathfrak{m})\left(p_{0}\right)=\mathfrak{g l}(m)$. Then by Sternberg's linearization theorem [4], there exist a vector field $X \in \mathfrak{m}$ and a smooth local coordinate system $\left(U ; x^{1}, \cdots, x^{m}\right)$ at $p_{0}$ such that $\left.X\right|_{U}=\sum_{i} x^{i}\left(\partial / \partial x^{i}\right)$. On the other hand, for any $Z \in \Gamma_{0}^{1}\left(T_{M}\right)$, there exists a sequence of neighborhoods $V \supset V_{1} \supset V_{2} \supset \cdots \supset V_{n} \supset \cdots$ of $p_{0}$ such that $V \subset U$ and

$$
Z=\sum_{i}\left(\sum_{|\alpha| \geq 2} \phi_{\alpha}^{i}(x) \cdot a_{\alpha}^{i} x^{\alpha}\right) \frac{\partial}{\partial x^{i}}+\tilde{Z} \quad \text { on } V \text {, }
$$

where $\phi_{\alpha}^{i}(x)$ is a $C^{\infty}$ function on $U$ such that

$$
\phi_{\alpha}^{i}(x)= \begin{cases}1 & \text { on } V_{i} \subset V \\ 0 & \text { outside some neighborhood of } V,\end{cases}
$$

$\sum_{|\alpha| \geqq 2} \phi_{\alpha}^{i}(x) \cdot a_{\alpha}^{i} \cdot x^{\alpha}$ is a power series which converges on $V$ and $\tilde{Z}$ is a $C^{\infty}$ vector field on $M$ such that $\tilde{Z}\left(p_{0}\right)=0$ and $j^{r}(\tilde{Z})\left(p_{0}\right)=0$ for all $r \geqq 1$ (see [4] p. 35). Now we consider the following power series

$$
\sum_{j}\left(\sum_{|\alpha| \geqq 2} \phi_{\alpha}^{j}(x) \cdot \frac{a_{\alpha}^{j}}{|\alpha|-1} \cdot x^{\alpha}\right) \cdot \frac{\partial}{\partial x^{j}} .
$$

This series converges on $V$ and becomes a $C^{\infty}$ vector field on $V$. Hence a suitable extension $Y$ of this vector field, i.e.

$$
\left.Y\right|_{V}=\sum_{j}\left(\sum_{|\alpha| \geqq 2} \phi_{\alpha}^{j}(x) \cdot \frac{a_{\alpha}^{j}}{|\alpha|-1} \cdot x^{\alpha}\right) \cdot \frac{\partial}{\partial x^{j}},
$$

is contained in $\Gamma_{0}^{1}\left(T_{M}\right)$. Since $X \in \mathfrak{m}$ and $\mathfrak{m}$ is an ideal of $\Gamma_{0}\left(T_{M}\right)$, we obtain $[X, Y] \in \mathfrak{m}$. Furthermore we have $[X, Y]=A^{j} \cdot \partial / \partial x^{j}$, where

$$
A^{j}=\sum_{i} x^{i}\left(\sum_{|\alpha| \geqq 2} \frac{\partial \phi_{\alpha}^{j}}{\partial x^{i}} \cdot \frac{a_{\alpha}^{j}}{|\alpha|-1} \cdot x^{\alpha}\right)+\sum_{|\alpha| \geqq 2} \phi_{\alpha}^{j} \cdot a_{\alpha}^{j} \cdot x^{\alpha} .
$$


By the definition of $\phi_{\alpha}^{j}$, we have

$$
\frac{\partial^{|\beta|} \phi_{\alpha}^{j}}{\partial x_{\beta}}=0 \quad \text { on } V_{j} \text {, for all multiple indices } \beta \text { with }|\beta| \geqq 1 \text {. }
$$

Therefore the Taylor expansion of $[X, Y]$ at $p_{0}=$ the Taylor expansion of $(Z-\tilde{Z})$ at $p_{0}$. Hence $Z-\tilde{Z}-[X, Y] \in \Gamma_{0}^{\infty}\left(T_{M}\right) \subset \mathfrak{m}$. Then $Z \in \mathfrak{m}$, hence $\Gamma_{0}^{1}\left(T_{M}\right) \subset \mathfrak{m}$. Therefore, by Proposition $2.8, \mathfrak{m}=\mathscr{I}_{z}$ or $\mathscr{I}_{s \ell}$. We have then $\mathfrak{f}^{1}(\mathfrak{m})\left(p_{0}\right) \subseteq \mathfrak{g l}(m)$, contradicting the assumption.

Proposition 2.11. If $\mathrm{m}$ is a maximal ideal of $\Gamma_{0}\left(T_{M}\right)$ such that $\mathfrak{m} \supset \boldsymbol{\Gamma}_{0}^{\infty}\left(T_{M}\right)$, then $\mathfrak{m}=\mathscr{I}_{z}$ or $\mathscr{I}_{s \ell}$.

Proof. By Lemma 2.10, $\dot{\mathfrak{f}}^{1}(\mathfrak{m})\left(p_{0}\right)$ is a proper ideal of $\mathrm{gl}(m)$. By Lemma 2.2, $\mathfrak{i}^{1}(\mathfrak{m})\left(p_{0}\right)$ should be equal to either $z$ or $s \ell(m)$. If $\mathfrak{j}^{1}(\mathfrak{m})\left(p_{0}\right)=z$ (resp. $s \ell(m)$ ), then $\mathfrak{m} \subset \mathscr{I}_{\mathfrak{z}}$ (resp. $\mathfrak{m} \subset \mathscr{I}_{s \ell}$ ). By the maximality of $\mathfrak{m}, \mathfrak{m}=$ $\mathscr{I}_{\mathrm{z}}\left(\right.$ resp. $\left.\mathfrak{m}=\mathscr{I}_{\mathrm{s} \ell}\right)$.

LEMMA 2.12. If $\mathfrak{m}$ is a maximal ideal of $\Gamma_{0}\left(T_{M}\right)$ such that $\mathfrak{m} \not \supset$ $\Gamma_{0}^{\infty}\left(T_{M}\right)$, then $\mathfrak{f}^{1}(\mathfrak{m})\left(p_{0}\right)=\mathfrak{g l}(m)$.

Proof. Assume $\mathfrak{j}^{1}(\mathfrak{m})\left(p_{0}\right)$ be a proper ideal of $\mathfrak{g l}(m)$. Then there occur three cases. If $\mathfrak{f}^{1}(\mathfrak{m})\left(p_{0}\right)=\{0\}$, then $\mathfrak{m} \subset \Gamma_{0}^{1}\left(T_{M}\right)$, contradicting the assumption. If $\mathfrak{j}^{1}(\mathfrak{m})\left(p_{0}\right)=z$ (resp. $\left.\mathfrak{i}^{1}(\mathfrak{m})\left(p_{0}\right)=s \ell(m)\right), \mathfrak{m} \supset \mathscr{I}_{z} \supset \Gamma_{0}^{\infty}\left(T_{M}\right)$ (resp. $\mathfrak{m} \supset \mathscr{I}_{s l} \supset \Gamma_{0}^{\infty}\left(T_{M}\right)$ ), contradicting the assumption. Hence $\mathfrak{i}^{1}(m)\left(p_{0}\right)$ should be equal to $\mathfrak{g l}(m)$.

LeMma 2.13. Let $\mathfrak{m}$ be a maximal ideal of $\Gamma_{0}\left(T_{M}\right)$ such that $\mathfrak{j}^{1}(\mathfrak{m})\left(p_{0}\right)$ $=g \mathfrak{l}(m)$. If for any $p \in M$ with $p \neq p_{0}$ there exists $Y \in \mathfrak{m}$ such that $Y(p) \neq 0$, then $\mathfrak{m} \supset \Gamma_{0}^{\infty}\left(T_{M}\right)$.

Proof. We set $\mathscr{I}_{p_{0}}^{C}=\left\{X \in \Gamma_{0}^{\infty}\left(T_{M}\right) \mid \operatorname{supp} X \not p_{0}\right\}$. First of all we prove that $\mathscr{I}_{p_{0}}^{C} \subset \mathrm{m}$.

(Remark that the assumption $\mathscr{I}_{p_{0}}^{C} \subset \mathfrak{m}$ has identical meaning with that of Lemma 1 of Pursell and Shanks [9], but unfortunately their proof contains a mistake about the argument of supports of the vector fields denoted by $N_{i}$. A complete proof for Lemma 1 is given in [5]. We use here the method used in [5].)

Let $X$ be an arbitrary element of $\mathscr{I}_{p_{0}}^{C}$. From the assumption of Lemma 2.13, for any $p \in \operatorname{supp} X$ there are a vector field $Y \in \mathfrak{m}$ and a local coordinate system $\left(V ; x^{1}, \cdots, x^{m}\right)$ such that $\left.Y\right|_{V}=\partial / \partial x^{1}$. Since 
supp $X$ is compact, there are $Y_{i} \in \mathfrak{m}, X_{i} \in \mathscr{I}_{p_{0}}^{c}$ and $\left(V_{i} ; x_{i}^{1}, \cdots, x_{i}^{m}\right), i=$ $1, \cdots, r$, such that $\left.Y_{i}\right|_{V_{i}}=\partial / \partial x_{i}^{1}, X=X_{1}+\cdots+X_{r}$, supp $X_{i} \subset V_{i}$ and $X_{i}=\sum_{k} \xi^{k}\left(\partial / \partial x_{i}^{k}\right)$ on $V_{i}$.

Hence if we want to prove that $X \in \mathfrak{m}$, it suffices to prove that $X_{i} \in \mathfrak{m}$ for each $i$. Because the argument is local we may delete the indices, that is, we may assume that there is a local coordinate system $\left(V ; x^{1}, \cdots, x^{m}\right)$ such that $X$ is written as $X=\sum \xi^{i}\left(\partial / \partial x^{i}\right)$ on $V$ with $\operatorname{supp} \xi^{i} \subset V$ for all $i=1, \cdots, m$, and a suitable extension of $\partial / \partial x^{1}$ is contained in $m$. We use the same notation for the extended vector fields because all argument here is local. Since $\partial / \partial x^{1} \in \mathfrak{m}$ and $\frac{1}{2}\left[\partial / \partial x^{1},\left(x^{1}\right)^{2}\left(\partial / \partial x^{1}\right)\right]$ $=x^{1}\left(\partial / \partial x^{1}\right), x^{1}\left(\partial / \partial x^{1}\right) \in \mathfrak{m}$. For $\xi^{1}\left(\partial / \partial x^{1}\right)$ we have the following formulae:

$$
\left[\frac{\partial}{\partial x^{1}}, x^{1} \xi^{1} \frac{\partial}{\partial x^{1}}\right]=\left(\xi^{1}+x^{1} \frac{\partial \xi^{1}}{\partial x^{1}}\right) \frac{\partial}{\partial x^{1}} \in \mathfrak{m}
$$

and

$$
\left[x^{1} \frac{\partial}{\partial x^{1}}, \xi^{1} \frac{\partial}{\partial x^{1}}\right]=\left(x^{1} \frac{\partial \xi^{1}}{\partial x^{1}}-\xi^{1}\right) \frac{\partial}{\partial x^{1}} \in \mathfrak{m}
$$

Hence we have $\frac{1}{2}\left(\left[\partial / \partial x^{1}, x^{1} \xi^{1}\left(\partial / \partial x^{1}\right)\right]-\left[x^{1}\left(\partial / \partial x^{1}\right), \xi^{1}\left(\partial / \partial x^{1}\right)\right]\right)=\xi^{1}\left(\partial / \partial x^{1}\right) \in \mathfrak{m}$. On the other hand for $\xi^{i}\left(\partial / \partial x^{i}\right), i \geqq 2$, we have the following formulae:

$$
\left[\frac{\partial}{\partial x^{1}}, x^{1} \xi^{i} \frac{\partial}{\partial x^{i}}\right]=\left(\xi^{i}+x^{1} \frac{\partial \xi^{i}}{\partial x^{1}}\right) \frac{\partial}{\partial x^{i}} \in \mathfrak{m}
$$

and

$$
\left[x^{1} \frac{\partial}{\partial x^{1}}, \xi^{i} \frac{\partial}{\partial x^{i}}\right]=x^{1} \frac{\partial \xi^{i}}{\partial x^{1}} \frac{\partial}{\partial x^{i}} \in \mathfrak{m} .
$$

Hence we have

$$
\left[\frac{\partial}{\partial x^{1}}, x^{1} \xi^{i} \frac{\partial}{\partial x^{i}}\right]-\left[x^{1} \frac{\partial}{\partial x^{1}}, \xi^{i} \frac{\partial}{\partial x^{i}}\right]=\xi^{i} \frac{\partial}{\partial x^{i}} \in \mathfrak{m} .
$$

Therefore we have $X=\sum \xi^{i}\left(\partial / \partial x^{i}\right) \in \mathfrak{m}$. Finally we obtain $\mathscr{I}_{p_{0}}^{C} \subset \mathfrak{m}$. Now we continue the proof of Lemma 2.13 .

Since $\dot{f}^{1}(\mathfrak{m})\left(p_{0}\right)=g l(m)$, by the Sternberg's linearization theorem there are a vector field $X \in \mathfrak{m}$ and a local coordidate system $\left(U ; x^{1}, \cdots, x^{m}\right)$ at $p_{0}$ such that $\left.X\right|_{U}=x^{i}\left(\partial / \partial x^{i}\right)$. For any $Z \in \Gamma_{0}^{\infty}\left(T_{M}\right)$ such that $\left.Z\right|_{U}=\zeta^{i}\left(\partial / \partial x^{i}\right)$ we consider the following system of differential equations on a neighborhood of $p_{0}$ : 


$$
x^{i} \frac{\partial \eta^{j}}{\partial x^{i}}-\eta^{j}=\zeta^{j} \quad(j=1, \cdots, m)
$$

By the polar coordinate system $x^{i}=r \phi_{i}\left(\theta^{1}, \cdots, \theta^{m-1}\right)(i=1, \cdots, m)$, above equations are written as

$$
r \frac{d \eta^{j}}{d r}-\eta^{j}=\zeta^{j} \quad(j=1, \cdots, m)
$$

where $r^{2}=\sum_{i}\left(x^{i}\right)^{2}$. By $r\left(d \eta^{j} / d r\right)-\eta^{j}=0$ we have $\eta^{j}=C(r) \cdot r$, where $C(r)$ is a function of $r$. So we have $d C / d r=\zeta^{j} / r^{2}$. Since $\zeta^{j}$ is flat at $r=0$,

$$
C(r)=\int_{0}^{r} \frac{\zeta^{j}}{r^{2}} d r
$$

Hence we have

$$
\eta^{j}=r \int_{0}^{r} \frac{\zeta^{j}}{r^{2}} d r
$$

on some neighborhood $W \subset U$ of $p_{0}$. Clearly $\eta^{j}(0)=0 \quad(j=1, \cdots, m)$. Therefore a suitable extension $Y$ of $\eta^{i}\left(\partial / \partial x^{i}\right)$, i.e. $\left.Y\right|_{W}=\eta^{i}\left(\partial / \partial x^{i}\right)$, is contained in $\Gamma_{0}\left(T_{M}\right)$. Obviously $\left.[X, Y]\right|_{W}=\left.Z\right|_{W}$. On the other hand $[X, Y] \in \mathfrak{m}$. We set $A=Z-[X, Y]$. Then $A \in \Gamma_{0}^{\infty}\left(T_{M}\right)$. Since $\operatorname{supp} A \nexists p_{0}, A \in \mathscr{I}_{p_{0}}^{C} \subset \mathfrak{m}$. Then $Z=A+[X, Y]$, hence $Z \in \mathfrak{m}$. Therefore $\Gamma_{0}^{\infty}\left(T_{M}\right) \subset \mathfrak{m}$.

Proposition 2.14. If $\mathfrak{m}$ is a maximal ideal of $\boldsymbol{\Gamma}_{0}\left(T_{M}\right)$ such that $\mathfrak{m} \downarrow \Gamma_{0}^{\infty}\left(T_{M}\right)$, then there exists a unique point $p \in M$ such that $p \neq p_{0}$ and $\mathfrak{m}=\mathscr{I}_{p}$.

Proof. By Lemma 2.12, $\mathfrak{i}^{1}(\mathfrak{m})\left(p_{0}\right)=g l(m)$. By Lemma 2.13, there exists a point $p \in M$ such that $p \neq p_{0}$ and $X(p)=0$ for all $X \in \mathfrak{m}$. By Lemma 2.4, $\mathfrak{m} \subset \mathscr{I}_{p}$. Since $\mathfrak{m}$ is a maximal ideal, $\mathfrak{m}=\mathscr{I}_{p}$. Furthermore the maximality of $\mathfrak{m}$ implies the uniqueness of the point $p$.

THEOREM 2.15. Any maximal ideal of $\Gamma_{0}\left(T_{M}\right)$ should be equal to one of the following ideals;

$\left.\begin{array}{ll}\text { (i ) } & \mathscr{I}_{z} \\ \text { (ii) } & \mathscr{I}_{\text {se }}\end{array}\right\}$ : ideal with finite codimension and corresponding to $p_{0}$,

(iii) $\mathscr{I}_{p}$ : ideal with infinite codimension and corresponding to $p$ $\left(p \neq p_{0}\right)$. 
Proof. The result is an immediate consequence of Propositions 2.11 and 2.14 .

§3. Characterization of maximal ideals of $\Gamma_{N}\left(T_{M}\right)(\operatorname{dim} N \geqq 1)$.

LEMMA 3.1. Let $X \in \Gamma_{N}\left(T_{M}\right)$ such that $X(p) \neq 0$ at $p \in M$. Then for any $Z \in \Gamma_{N}\left(T_{M}\right)$ there exist an element $Y \in \Gamma_{N}\left(T_{M}\right)$ and a neighborhood $U$ of $p$ in $M$ such that $[X, Y]=Z$ on $U$.

Proof. The case $p \notin N$ was already proved in Lemma 2.1. Let $p$ be a point in $N$. By Lemma 1.5 we can take a local coordinate system $\left(U ; x^{1}, \cdots, x^{n}, y^{1}, \cdots, y^{\ell}\right)$ at $p$ such that $U \cap N=\left\{y^{1}=\cdots=y^{\ell}=0\right\}$ and $X=\partial / \partial x^{1}$ on $U$. For any $Z=\zeta^{i}\left(\partial / \partial x^{i}\right)+\zeta^{\alpha}\left(\partial / \partial y^{\alpha}\right) \in \Gamma_{N}\left(T_{M}\right)$ we consider the following differetial equations.

$$
\begin{cases}\frac{\partial \eta^{i}}{\partial x^{1}}=\zeta^{i} & (i=1, \cdots, n) \\ \frac{\partial \eta^{\alpha}}{\partial x^{1}}=\zeta^{\alpha} & (\alpha=1, \cdots, \ell), \text { where } \zeta^{\alpha}\left(x^{1}, \cdots, x^{n}, 0, \cdots, 0\right)=0 .\end{cases}
$$

These equations have solutions on $U$ :

$$
\left\{\begin{array}{l}
\eta^{i}=\int \zeta^{i} d x^{1}+C^{i}\left(x^{2}, \cdots, x^{n}, y^{1}, \cdots, y^{\ell}\right) \\
\eta^{\alpha}=\int \zeta^{\alpha} d x^{1}+C^{\alpha}\left(x^{2}, \cdots, x^{n}, y^{1}, \cdots, y^{\ell}\right)
\end{array}\right.
$$

Set $C^{\alpha}\left(x^{2}, \cdots, x^{n}, 0, \cdots, 0\right)=0$ for $\alpha=1, \cdots, \ell$.

Then $\eta^{\alpha}\left(x^{1}, \cdots, x^{n}, 0, \cdots, 0\right)=0$. Let $Y$ be an appropriate extension of $\eta^{i}\left(\partial / \partial x^{i}\right)+\eta^{\alpha}\left(\partial / \partial y^{\alpha}\right)$. Then $Y \in \Gamma_{N}\left(T_{M}\right)$ and $[X, Y]=Z$ on $U$.

LEMMA 3.2. For any proper ideal $\mathscr{I} \subset \Gamma_{N}\left(T_{M}\right)$ there exists a point $p \in M$ such that $X(p)=0$ for all $X \in \mathscr{I}$.

Proof. The proof is done by the method which was used to prove $\mathscr{I}_{p_{0}}^{C} \subset \mathfrak{m}$ in Lemma 2.13, and omitted.

LeMma 3.3. Let $\mathscr{I} \subseteq \Gamma_{N}\left(T_{M}\right)$ be an ideal, and $p \in M$ be a point such that $X(p)=0$ for all $X \in \mathscr{I}$.

(Case $p \notin N)$ Let $\left(U ; x^{1}, \cdots, x^{m}\right)$ be a local coordinate system at $p$. Then for any $X=\xi^{i}\left(\partial / \partial x^{i}\right) \in \mathscr{I}$ we have

$$
\frac{\partial^{r} \xi^{i}}{\partial x^{i_{1}} \cdots \partial x^{i_{r}}}(p)=0 \quad(1 \leqq i \leqq m ; 1 \leqq r)
$$


(Case $p \in N)$ Let $\left(U ; x^{1}, \cdots, x^{n}, y^{\ell} \cdots, y^{l}\right)$ be a local coordinate system at $p$ in $M$ such that $U \cap N=\left\{y^{1}=\cdots=y^{\ell}=0\right\}$.

Then for any $X=\xi^{i}\left(\partial / \partial x^{i}\right)+\xi^{\alpha}\left(\partial / \partial y^{\alpha}\right) \in \mathscr{I}$ we have

$$
\begin{aligned}
\frac{\partial^{r} \xi^{i}}{\partial x^{i_{1}} \cdots \partial x^{i_{r}}}(p)=0 \text { and } & \frac{\partial^{r} \xi^{\alpha}}{\partial x^{i_{1}} \cdots \partial x^{i_{r}}}(p)=0 \\
& (1 \leqq i \leqq n ; 1 \leqq \alpha \leqq \ell ; 1 \leqq r) .
\end{aligned}
$$

Proof. The proof is all the same as that of Lemma 2.4.

LEMMA 3.4. Let $\mathscr{I}$ be a proper ideal of $\Gamma_{N}\left(T_{M}\right)$ such that $X(p)=0$ for all $X \in \mathscr{I}$ at a point $p \in M$. Then $p \notin N$, if and only if $\mathscr{I}$ does not contain $\operatorname{Ker} r_{*}$, where $r_{*}: \Gamma_{N}\left(T_{M}\right) \rightarrow \Gamma\left(T_{N}\right)$ is the Lie algebra homomorphism.

Proof. Easy computation.

Let $p$ be a point of $M$. We denote by $\mathscr{I}_{p}$ the ideal of $\Gamma_{N}\left(T_{M}\right)$ consisting of all element $X$ such that $X$ and its all derivatives vanish at the point $p$. Clearly if $p \notin N$, then $\mathscr{I}_{p}$ is a maximal ideal of $\Gamma_{N}\left(T_{M}\right)$.

For a given point $p \in N$ we denote by $\overline{\mathscr{I}}_{p}$ the ideal of $\Gamma\left(T_{N}\right)$ consisting of all element $Y$ such that $Y$ and its all derivatives vanish at the point $p . \quad \overline{\mathscr{I}}_{p}$ is a maximal ideal of $\Gamma\left(T_{N}\right)$.

Proposition 3.5. For any maximal ideal $\mathscr{I}$ of $\Gamma_{N}\left(T_{M}\right)$, there exists a unique point $p \in M$ such that

$$
\mathscr{I}= \begin{cases}\mathscr{I}_{p} & \left(\text { if } \mathscr{I} \text { does not contain } \operatorname{Ker} r_{*}\right) \\ r_{*}^{-1} \overline{\mathscr{I}}_{p} & \left(\text { if } \mathscr{I} \text { contains } \operatorname{Ker} r_{*}\right) .\end{cases}
$$

Proof. By Lemma 3.2 there is a point $p \in M$ such that $X(p)=0$ for all $X \in \mathscr{I}$. If $\mathscr{I}$ does not contain $\operatorname{Ker} r_{*}$, then $p$ is never contained in $N$. Hence by Lemma $3.3 \mathscr{I}$ is contained in the proper ideal $\mathscr{I}_{p}$. Since $\mathscr{I}$ is maximal, $\mathscr{I}=\mathscr{I}_{p}$. If $\mathscr{I}$ contains $\operatorname{Ker} r_{*}$, by Lemma 3.3 $r_{*}(\mathscr{I}) \subset \overline{\mathscr{I}}_{p}$. By the maximality of $\mathscr{I}, r_{*}(\mathscr{I})$ is also maximal in $\Gamma\left(T_{N}\right)$. Hence $r_{*}(\mathscr{I})=\overline{\mathscr{I}}_{p}$. Therefore $\mathscr{I}=r_{*}^{-1} \bar{I}_{p}$. Furthermore the maximality of $\mathscr{I}$ implies the uniqueness of the point $p$.

LEMMA 3.6. $\quad \Gamma_{N}\left(T_{M}\right) / \mathscr{I}_{p} \cong R[\left[x^{1}, \cdots, x^{m}\right] \overbrace{\times \cdots \times R}^{m}\left[\left[x^{1}, \cdots, x^{m}\right]\right]$ and $\Gamma_{N}\left(T_{M}\right) / r_{*}^{-1} \bar{I}_{p} \cong R[\left[x^{1}, \cdots, x^{n}\right] \overbrace{\times \cdots \times R}^{n}\left[\left[x^{1}, \cdots, x^{n}\right]\right]$ as Lie algebras, where $m=n+\ell$ and $R[[\cdots]]$ is the ring of formal power series. 
Proof. Let $\left(U ; x^{1}, \cdots, x^{m}\right)$ be a local coordinate system at $p \in M$. Then the formal Taylor expansion of $X \in \Gamma_{N}\left(T_{M}\right)$ at $p$ with respect to this coordinate is a homomorphism of $\Gamma_{N}\left(T_{M}\right)$ onto the product of the rings of formal power series, and its kernel is exactly $\mathscr{I}_{p}$.

For the case $p \in N$ we consider the following commutative diagram:

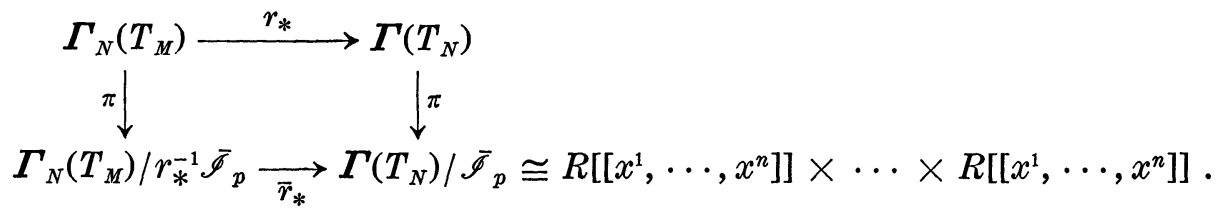

Since $\bar{r}_{*}$ is an isomorphism, we have the desired result.

\section{§ 4. Stone topology of maximal ideal sets.}

(Case $\Gamma_{0}\left(T_{M}\right)$ ) Let $M$ and $M^{\prime}$ be $C^{\infty}$ manifolds and $p_{0}$ (resp. $p_{0}^{\prime}$ ) be an arbitrary but fixed point of $M$ (resp. $\left.M^{\prime}\right)$. We define $\Gamma_{0}\left(T_{M}\right), \Gamma_{0}\left(T_{M^{\prime}}\right)$, $\Gamma_{0}^{1}\left(T_{M}\right)$ and $\Gamma_{0}^{1}\left(T_{M^{\prime}}\right)$ as in $\S 2$.

LEMMA 4.1. If $\Phi: \Gamma_{0}\left(T_{M}\right) \rightarrow \Gamma_{0}\left(T_{M^{\prime}}\right)$ is a Lie algebra isomorphism, then $\Phi\left(\mathscr{I}_{z}\right)=\mathscr{I}_{z^{\prime}}, \Phi\left(\mathscr{I}_{s \ell}\right)=\mathscr{I}_{s \ell^{\prime}}$ and $\Phi\left(\mathscr{I}_{p}\right)=\mathscr{I}_{p^{\prime}}\left(\right.$ if $\left.p \neq p_{0}\right)$. Especially $\Phi\left(\Gamma_{0}^{1}\left(T_{M}\right)\right)=\Gamma_{0}^{1}\left(T_{M^{\prime}}\right)$.

Proof. If $\mathfrak{m}^{\prime}=\Phi(\mathfrak{m})$ is a maximal ideal, then $\Gamma_{0}\left(T_{M}\right) / \mathfrak{m}$ is isomorphic to $\Gamma_{0}\left(T_{M^{\prime}}\right) / \mathfrak{m}^{\prime}$. Hence codim $\mathfrak{m}$ in $\Gamma_{0}\left(T_{M}\right)=\operatorname{codim} \mathfrak{m}^{\prime}$ in $\Gamma_{0}\left(T_{M^{\prime}}\right)$. Since $\operatorname{codim} \mathscr{I}_{z}=n^{2}-1$ and codim $\mathscr{I}_{s \ell}=1$, we have $\Phi\left(\mathscr{I}_{g}\right)=\mathscr{I}_{z^{\prime}}$ and $\Phi\left(\mathscr{I}_{s \ell}\right)=\mathscr{I}_{s \ell^{\prime}}$. On the other hand, since each ideal $\mathscr{I}_{p}$ which has infinite codimension corresponds to a point $p\left(p \neq p_{0}\right)$ uniquely, $\Phi\left(\mathscr{I}_{p}\right)=\mathscr{I}_{p^{\prime}}$ for some unique point $p^{\prime}\left(p^{\prime} \neq p_{0}^{\prime}\right)$. Moreover, since $\Gamma_{0}^{1}\left(T_{M}\right)=\mathscr{I}_{z} \cap \mathscr{I}_{\text {se }}$, $\Phi\left(\Gamma_{0}^{1}\left(T_{M}\right)\right)=\Gamma_{0}^{1}\left(T_{M^{\prime}}\right)$.

We denote by $M^{*}$ the set of all maximal ideals of $\Gamma_{0}\left(T_{M}\right)$, that is,

$$
M^{*}=\left\{\mathscr{I} \mid \mathscr{I} \subset \Gamma_{0}\left(T_{M}\right): \text { maximal ideal }\right\} \text {. }
$$

From now on, we denote both $\mathscr{I}_{z}$ and $\mathscr{I}_{s \ell}$ simply $\mathscr{I}_{p_{0}}$. Let $\sigma: M^{*} \rightarrow M$ be the natural correspondence defined by $\sigma\left(\mathscr{I}_{p}\right)=p$. (Note. $\sigma\left(\mathscr{I}_{p_{0}}\right)=$ $\left.\sigma\left(\mathscr{I}_{z}\right)=\sigma\left(\mathscr{I}_{s \ell}\right)=p_{0}\right)$

For any subset $A \subset M$ we set $A^{*}=\sigma^{-1}(A)=\left\{\mathscr{I}_{p} \in M^{*} \mid p \in A\right\}$. .

Definition 4.2. (Stone topology of $M^{*}$ ) For any subset of $M^{*}$ we define a closure operator " $\mathscr{l} l$ " by the formulas: 
(i) $\mathscr{C} \ell \phi=\phi$

(ii) If $B \neq \phi$ then $\mathscr{C} \ell B=\{\mathfrak{m} \mid \mathfrak{m}$ is a maximal ideal such that $\left.\mathrm{m} \supset \bigcap_{s \in B} \mathscr{I}\right\}$.

DEFINITION 4.3. We call a subset $B \subset M^{*}$ is closed, if and only if $\mathscr{C} \ell B=B$.

LEMMA 4.4. For each $A^{*}=\sigma^{-1}(A), \mathscr{C} \ell\left(A^{*}\right)=(\bar{A})^{*}$, where $\bar{A}$ is the closure of $A$ in $M$.

Proof. First, we prove " $\subset$ ". For any $\mathfrak{m} \in \mathscr{C} \ell\left(A^{*}\right)$, since $\mathfrak{m}$ is a maximal ideal, there exists a unique point $p \in M$ such that $\mathfrak{m}=\mathscr{I}_{p}$ (may be $p=p_{0}$ ). Assume $p \notin \bar{A}$.

(Case $\left.p \neq p_{0}\right) \quad \mathfrak{m}=\mathscr{I}_{p} \supset \bigcap_{\kappa \in A^{*}} \mathscr{I}$. On the other hand, since $p \notin \bar{A}$, there is $X \in \cup_{s \in A^{*}} \mathscr{I}$ such that $X(p) \neq 0$. Hence $X \notin \mathscr{I}_{p} \ldots$ contradiction.

(Case $p=p_{0}$ ) There are two cases, one is $\mathfrak{m}=\mathscr{I}_{z} \supset \bigcap_{s \in A^{*}} \mathscr{I}$ and other is $\mathfrak{m}=\mathscr{I}_{s \ell} \supset \bigcap_{s \in A^{*}} \mathscr{I}$. On the other hand there is $Y \in \Gamma_{0}\left(T_{M}\right)$ such that $j^{1}(Y)\left(p_{0}\right) \notin z \cup s \ell(m)$ (set union). Let $\psi: M \rightarrow R$ be a $C^{\infty}$ function such that

$$
\psi= \begin{cases}1 & \text { in some neighborhood } U \text { of } p_{0} \text { with } U \cap \bar{A}=\phi \\ 0 & \text { outside some neighborhood of } U .\end{cases}
$$

Then $X=\psi Y \in \bigcap_{s \in A^{*}} \mathscr{I}$ and $j^{1}(X)\left(p_{0}\right) \notin z \cup s \ell(m)$, that is, $X \notin \mathscr{I}_{z} \cup \mathscr{I}_{s \ell} \ldots$ contradiction. Therefore $p$ should be contained in $\bar{A}$. So $\mathfrak{m} \in(\bar{A})^{*}$.

Next we prove “ $\supset$ ". For any $\mathscr{I}_{p} \in(\bar{A}) *$ (may be $p=p_{0}$ ), $p \in \bar{A}$. If $p \in A$, then clearly $\mathscr{I}_{p} \in \mathscr{C} \ell\left(A^{*}\right)$. So we may assume $p \in \bar{A}-A$. For any $Y \in \bigcap_{s \in A^{*}} \mathscr{I}, Y=0$ on $A$. Since $Y$ is a $C^{\infty}$ vector field, $Y(p)=0$ and $\mathrm{j}^{r}(Y)(p)=0$ for all $r \geqq 1$. Hence $Y \in \mathscr{I}_{p}$ (may be $p=p_{0}$ ). Therefore $\mathscr{I}_{p} \supset \bigcap_{s \in A^{*}} \mathscr{I}$, that is, $\mathscr{I}_{p} \in \mathscr{C} \ell\left(A^{*}\right)$. This completes the proof of Lemma 4.4 .

LEMMA 4.5. The natural correspondence $\sigma: M^{*} \rightarrow M$ preserves the concept of closed subsets defined by Definition 4.3, that is, $A$ is a closed subset of $M$, if and only if $A^{*}=\sigma^{-1}(A)$ is a closed subset of $M^{*}$.

Proof. Let $A$ be a closed subset of $M$. By Lemma 4.4, $\mathscr{C} \ell\left(A^{*}\right)=$ $(\bar{A})^{*}=A^{*}$. Hence $A^{*}$ is closed.

Conversely, let $A^{*}=\sigma^{-1}(A)$ be a closed subset of $M^{*}$, then by Lemma $4.4,(\bar{A})^{*}=\mathscr{C} \ell\left(A^{*}\right)=A^{*}$. Hence $\bar{A}=\sigma\left((\bar{A})^{*}\right)=\sigma\left(A^{*}\right)=A$. So $A$ is closed. 
LEMMA 4.6. Let $\Phi: \Gamma_{0}\left(T_{M}\right) \rightarrow \Gamma_{0}\left(T_{M}\right)$ be a Lie algebra isomorphism. Then $A^{*}$ is a closed subset of $M^{*}$, if and only if $\Phi\left(A^{*}\right)$ is a closed subset of $\left(M^{\prime}\right)^{*}$, where $\Phi\left(A^{*}\right)=\left\{\Phi(\mathscr{I}) \mid \mathscr{I} \in A^{*}\right\}$.

Proof. Since $\Phi$ is an isomorphism, $\Phi: M^{*} \rightarrow\left(M^{\prime}\right)^{*}$ is a one to one, onto correspondence. So we have

$$
\Phi\left(\bigcap_{s \in A^{*}} \mathscr{I}\right)=\bigcap_{s \in A^{*}} \Phi(\mathscr{I})=\bigcap_{\mathscr{s}^{\prime} \in \Phi\left(A^{*}\right)} \mathscr{I}^{\prime} .
$$

Hence we have $\mathfrak{m} \supset \bigcap_{\mathscr{s} \in A^{*}} \mathscr{I}$, if and only if $\Phi(\mathfrak{m}) \supset \bigcap_{\mathscr{N}^{\prime} \in \mathscr{(}\left(A^{*}\right)} \mathscr{I}^{\prime}$. This completes the proof of Lemma 4.6.

Now we define a map $\varphi: M \rightarrow M^{\prime}$ by the following formula.

$$
\left\{\begin{array}{l}
\varphi\left(p_{0}\right)=p_{0}^{\prime} \\
\varphi(p)=p^{\prime}, \quad \text { if } p \neq p_{0} \text { and } \Phi\left(\mathscr{I}_{p}\right)=\mathscr{I}_{p^{\prime}} .
\end{array}\right.
$$

Proposition 4.7. The natural map $\varphi: M \rightarrow M^{\prime}$ is an onto homeomorphism.

Proof. Clearly $\varphi$ is a one to one and onto map. From the definition of $\varphi$, we have the following commutative diagram.

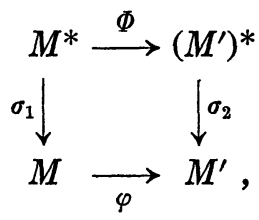

where $\sigma_{i}$ is the natural correspondence. Let $B$ be an arbitrary closed subset of $M^{\prime}$. By Lemmas 4.5 and 4.6, $\left(\Phi^{-1} \circ \sigma_{2}^{-1}\right)(B)$ is a closed subset of $M^{*}$. Since $\sigma_{1}^{-1}\left(\varphi^{-1}(B)\right)=\left(\Phi^{-1} \circ \sigma_{2}^{-1}\right)(B)$, we see by Lemma 4.5 that $\varphi^{-1}(B)$ is a closed subset of $M$. Hence $\varphi$ is a continuous map. By the same way we can prove that $\varphi^{-1}$ is also continuous. Hence $\varphi$ is a homeomorphism.

Next we study the case $\Gamma_{N}\left(T_{M}\right)$ with $\operatorname{dim} N \geqq 1$. Let $M$ and $M^{\prime}$ be $C^{\infty}$ manifolds and $N$ (resp. $N^{\prime}$ ) be an arbitrary but fixed closed submanifold of $M$ (resp. $\left.M^{\prime}\right)$.

Proposition 4.8. Let $\Phi: \Gamma_{N}\left(T_{M}\right) \rightarrow \Gamma_{N^{\prime}}\left(T_{M^{\prime}}\right)$ be an isomorphism. Let $\mathscr{I}$ be the maximal ideal of $\Gamma_{N}\left(T_{M}\right)$ corresponding to $p$, and $\mathscr{I}^{\prime} \doteq \Phi(\mathscr{I})$ be the maximal ideal of $\Gamma_{N^{\prime}}\left(T_{M^{\prime}}\right)$ corresponding to $p^{\prime}$. Then $p \in N$, if and only if $p^{\prime} \in N^{\prime}$. 
Proof. Since $\Phi: \Gamma_{N}\left(T_{M}\right) \rightarrow \Gamma_{N^{\prime}}\left(T_{M^{\prime}}\right)$ is an isomorphism, $\Gamma_{N}\left(T_{M}\right) / \mathscr{I}$ should be isomorphic to $\Gamma_{N^{\prime}}\left(T_{M^{\prime}}\right) / \mathscr{I}^{\prime}$. By Lemma 3.6 this implies $p \in N$ $\Leftrightarrow p^{\prime} \in N^{\prime}$.

LEMMA 4.9. Let $\Phi: \Gamma_{N}\left(T_{M}\right) \rightarrow \Gamma_{N^{\prime}}\left(T_{M^{\prime}}\right)$ be an isomorphism. Then $\Phi\left(\operatorname{Ker} r_{*}\right)=\operatorname{Ker} r_{*}^{\prime}$, that is, $\Phi$ induces an isomorphism $\Psi: \Gamma\left(T_{N}\right) \rightarrow \Gamma\left(T_{N^{\prime}}\right)$, where $r_{*}: \Gamma_{N}\left(T_{M}\right) \rightarrow \Gamma\left(T_{N}\right)$ (resp. $r_{*}^{\prime}: \Gamma_{N^{\prime}}\left(T_{M^{\prime}}\right) \rightarrow \Gamma\left(T_{N^{\prime}}\right)$ ) is the homomorphism induced by the restriction of vector fields on $M$ (resp. $M^{\prime}$ ) to $N$ (resp. $N^{\prime}$ ).

Proof. Obviously $\operatorname{Ker} r_{*}=\bigcap\left\{r_{*}^{-1} \overline{\mathscr{I}}_{p} \mid p \in N\right\}$. By Proposition 4.8, $\Phi\left(\operatorname{Ker} r_{*}\right)=\bigcap\left\{\Phi\left(r_{*}^{-1} \bar{I}_{p}\right) \mid p \in N\right\}=\bigcap\left\{r_{*}^{\prime-1} \bar{I}_{p} \mid q \in N^{\prime}\right\}=\operatorname{Ker} r_{*}^{\prime}$.

Let $\Phi: \Gamma_{N}\left(T_{M}\right) \rightarrow \Gamma_{N^{\prime}}\left(T_{M^{\prime}}\right)$ be an isomorphism. Let $\mathscr{I}$ be the maximal ideal corresponding to $p \in M$. Then by Proposition 3.5 there exists a unique point $q \in M^{\prime}$ such that the maximal ideal $\mathscr{I}^{\prime}=\Phi(\mathscr{I})$ corresponds to $q$. We set $\varphi(p)=q$. Now we define the Stone topology of $M^{*}=$ $\left\{\mathscr{I} \mid \mathscr{I} \subset \Gamma_{N}\left(T_{M}\right):\right.$ maximal ideal $\}$ as in the case $\Gamma_{0}\left(T_{M}\right)$. Then we have the following proposition.

Proposition 4.10. The natural correspondence $\varphi: M \rightarrow M^{\prime}$ is an onto homeomorphism such that $\varphi(N)=N^{\prime}$.

Proof. The proof for $\varphi$ to be a homeomorphism is all the same as that of the case $\Gamma_{0}\left(T_{M}\right)$. By Proposition 4.8, $\varphi(N)=N^{\prime}$.

\section{§ 5. Characterization of non-zero vector fields.}

LEMMA 5.1. Let $\mathscr{I}_{p}$ be the maximal ideal of $\Gamma_{0}\left(T_{M}\right)$ corresponding to $p\left(p \neq p_{0}\right)$. For any $X \in \Gamma_{0}\left(T_{M}\right), X(p) \neq 0$, if and only if $\left[X, \Gamma_{0}\left(T_{M}\right)\right]$ $+\mathscr{I}_{p}=\Gamma_{0}\left(T_{M}\right)$.

LEMMA 5.1'. For any $X \in \Gamma_{N}\left(T_{M}\right), X(p) \neq 0$, if and only if

(i) $\left[X, \Gamma_{N}\left(T_{M}\right)\right]+\mathscr{I}_{p}=\Gamma_{N}\left(T_{M}\right)($ for $p \notin N)$ or

(ii) $\left[r_{*} X, \Gamma\left(T_{N}\right)\right]+\overline{\mathscr{I}}_{p}=\Gamma\left(T_{N}\right)($ for $p \in N)$.

Proof. The proofs of these lemmas are all the same as that of Lemma 3 of Pursell and Shanks [9] (see also Omori (5]), and omitted.

LEMMA 5.2. Let $\Phi: \Gamma_{0}\left(T_{M}\right) \rightarrow \Gamma_{0}\left(T_{M^{\prime}}\right)$ be a Lie algebra isomorphism and $\varphi: M \rightarrow M^{\prime}$ be the induced homeomorphism. For any $p \in M\left(p \neq p_{0}\right)$ there are smooth local coordinate system $\left(U ; x^{1}, \cdots, x^{m}\right)$ at $p$ and $\left(V ; y^{1}\right.$, $\left.\cdots, y^{m}\right)$ at $\varphi(p)=p^{\prime}$ such that for any 


$$
X=\xi^{i} \frac{\partial}{\partial x^{i}} \in \Gamma_{0}\left(T_{M}\right), \quad \Phi\left(\xi^{i} \frac{\partial}{\partial x^{i}}\right)=\left(\xi^{i} \circ \varphi^{-1}\right) \frac{\partial}{\partial y^{i}}
$$

Proof. Since $p \neq p_{0}$, there is a smooth local coordinate system ( $U$; $\left.x^{1}, \cdots, x^{m}\right)$ at $p$ such that $p_{0} \notin \bar{U}$. Hence suitable extensions of $\partial / \partial x^{i}(i=$ $1, \cdots, m)$ are contained in $\Gamma_{0}\left(T_{M}\right)$. We also denote the extended vector fields by the same letters. Set $v_{i}=\Phi\left(\partial / \partial x^{i}\right) \quad(i=1, \cdots, m)$. Then $v_{i} \in \Gamma_{0}\left(T_{M^{\prime}}\right)$ for all $i=1, \cdots, m$. Since $\left(\partial / \partial x^{i}\right)(p) \neq 0$, by Lemma 5.1, $v_{i}\left(p^{\prime}\right) \neq 0$, where $p^{\prime}=\varphi(p)$. Since $\Phi$ is a Lie algebra isomorphism, on some neighborhood of $p^{\prime},\left[v_{i}, v_{j}\right]=\Phi\left(\left[\partial / \partial x^{i}, \partial / \partial x^{j}\right]\right)=0$ for all $i, j=1, \cdots$, $m$. Hence there exists a smooth local coordinate system $\left(V ; y^{1}, \cdots, y^{m}\right)$ at $p^{\prime}$ such that $v_{i}=\partial / \partial y^{i}$ on $V$. Let $q$ be an arbitrary point in $U$. Now, for any $X=\xi^{i}\left(\partial / \partial x^{i}\right) \in \Gamma_{0}\left(T_{M}\right)$, a suitable extension of $\xi^{i}(q)\left(\partial / \partial x^{i}\right)$ is contained in $\Gamma_{0}\left(T_{M}\right)$. We denote it by $X^{*}$. Since $\left(X-X^{*}\right)(q)=0$, by Lemma 5.1, $\Phi\left(X-X^{*}\right)\left(q^{\prime}\right)=0$. Hence $\Phi(X)\left(q^{\prime}\right)=\Phi\left(X^{*}\right)\left(q^{\prime}\right)=\xi^{i}(q) \cdot v_{i}\left(q^{\prime}\right)$ $=\left(\xi^{i} \circ \varphi^{-1}\left(q^{\prime}\right)\right) \cdot\left(\partial / \partial y^{i}\right)\left(q^{\prime}\right)$. Therefore $\Phi\left(\xi^{i}\left(\partial / \partial x^{i}\right)\right)=\left(\xi^{i} \circ \varphi^{-1}\right)\left(\partial / \partial y^{i}\right)$ on $V$.

COROLLARY 5.3. The induced homeomorphism $\varphi: M \rightarrow M^{\prime}$ is linear with respect to the local coordinate systems defined in Lemma 5.2 , that $i s, \varphi^{i}\left(x^{1}, \cdots, x^{m}\right)=x^{i}(i=1, \cdots, m)$, where $\varphi^{i}=y^{i} \circ \varphi$.

Proof. We use the same notations for the extended vector fields because all argument here is local. By Lemma 5.2, $\Phi\left(x^{i}\left(\partial / \partial x^{j}\right)\right)=$ $\left(x^{i} \circ \varphi^{-1}\right)\left(\partial / \partial y^{j}\right)$. On the other hand we have $\left[\partial / \partial y^{k},\left(x^{i} \circ \varphi^{-1}\right)\left(\partial / \partial y^{j}\right)\right]=$ $\left(\partial / \partial y^{k}\right)\left(x^{i} \circ \varphi^{-1}\right)\left(\partial / \partial y^{j}\right)$ and $\left[\partial / \partial y^{k},\left(x^{i} \circ \varphi^{-1}\right)\left(\partial / \partial y^{j}\right)\right]=\Phi\left(\left[\partial / \partial x^{k}, x^{i}\left(\partial / \partial x^{j}\right)\right]\right)=$ $\delta_{k}^{i}\left(\partial / \partial y^{j}\right)$, where $\delta_{k}^{i}$ is the Kronecker delta. So we have $\left(\partial / \partial y^{k}\right)\left(x^{i} \circ \varphi^{-1}\right)=$ $\delta_{k}^{i}$. Hence $x^{i} \circ \varphi^{-1}=y^{i}+C$, where $C$ is a constant of integration. Since $\varphi(0)=0, C=0$. Therefore $x^{i} \circ \varphi^{-1}=y^{i}$. Since $\varphi$ is a homeomorphism, $y^{i} \circ \varphi=\left(x^{i} \circ \varphi^{-1}\right) \circ \varphi=x^{i}$.

Proposition 5.4. Let $\Phi: \Gamma_{0}\left(T_{M}\right) \rightarrow \Gamma_{0}\left(T_{M^{\prime}}\right)$ be a Lie algebra isomorphism and $\varphi: M \rightarrow M^{\prime}$ be the induced homeomorphism. Then $\left.\varphi\right|_{M_{0}}: M_{0}$ $\rightarrow M_{0}^{\prime}$ is a $C^{\infty}$ diffeomorphism, where $M_{0}=M-\left\{p_{0}\right\}$ and $M_{0}^{\prime}=M^{\prime}-\left\{p_{0}^{\prime}\right\}$.

Proof. Let $f \in C^{\infty}\left(M_{0}\right)$ be an arbitrary $C^{\infty}$ function of $M_{0}$ and $p^{\prime} \in M_{0}^{\prime}$ be an arbitrary point, and set $p^{\prime}=\varphi(p)$. Let $\left(U ; x^{1}, \cdots, x^{m}\right)$ be a local coordinate system at $p$ in $M_{0}$. Since $p_{0} \notin U$, a suitable extension of $f \cdot \partial / \partial x^{1}$ is contained in $\Gamma_{0}\left(T_{M}\right)$. We denote the extended vector field by the same letter. By Lemma 5.2, $\Phi\left(f \cdot \partial / \partial x^{1}\right)=\left(f \circ \varphi^{-1}\right) \cdot \partial / \partial y^{1}$ on some coordinate neighborhood $V$ of $p^{\prime} \in M_{0}^{\prime}$. Since $\Phi\left(f \cdot \partial / \partial x^{1}\right)$ is a $C^{\infty}$ vector 
field, $f \circ \varphi^{-1}$ is a $C^{\infty}$ function on $V$. Since $p^{\prime}$ and $f$ are arbitrary, $\varphi$ is a diffeomorphism.

COROLlaRY 5.5. Let $\varphi: M \rightarrow M^{\prime}$ be the homeomorphism induced by the isomorphism $\Phi$. Then $\Phi=d \varphi$ on $M-\left\{p_{0}\right\}$.

Proof. For each point $p \in M-\left\{p_{0}\right\}$, by Lemma 5.2 and Corollary $5.3 \varphi_{i}\left(x^{1}, \cdots, x^{m}\right)=y^{i} \circ \varphi\left(x^{1}, \cdots, x^{m}\right)=x^{i}$ in some neighborhood of $p$. Hence for any $X=\xi^{i}\left(\partial / \partial x^{i}\right) \in \Gamma_{0}\left(T_{M}\right)$ we have

$$
d \varphi(X)=\left(\xi^{i} \circ \varphi^{-1}\right)\left(\frac{\partial \varphi_{j}}{\partial x^{i}}\right) \cdot \frac{\partial}{\partial y^{j}}=\left(\xi^{i} \circ \varphi^{-1}\right) \cdot \frac{\partial}{\partial y^{i}} .
$$

On the other hand $\Phi(X)=\left(\xi^{i} \circ \varphi^{-1}\right) \partial / \partial y^{i}$. Hence $d \varphi=\Phi$ on $M-\left\{p_{0}\right\}$.

\section{\$ 6. Proof of the theorem.}

(Case $\Gamma_{.0}\left(T_{M}\right)$ )

LEMmA 6.1. For any $Y \in \Gamma_{0}\left(T_{M^{\prime}}\right)$ and any $g \in C^{\infty}\left(M^{\prime}\right)$ we have

$$
\Phi^{-1}(g Y)=(g \circ \varphi) \Phi^{-1}(Y) \text {. }
$$

Proof. For the case $p \neq p_{0}$ we already proved in Lemma 5.2. Set $Z=g Y-g\left(p_{0}^{\prime}\right) \cdot Y$, where $p_{0}^{\prime}=\varphi\left(p_{0}\right)$. Clearly $Z\left(p_{0}^{\prime}\right)=0$. Since $\Phi^{-1}: \Gamma_{0}\left(T_{M^{\prime}}\right)$ $\rightarrow \Gamma_{0}\left(T_{M}\right)$ is an isomorphism, $\Phi^{-1}(0)=0$. Hence $\Phi^{-1}(Z)\left(p_{0}\right)=\Phi^{-1}(g Y)\left(p_{0}\right)$ $-g\left(p_{0}^{\prime}\right) \Phi^{-1}(Y)\left(p_{0}\right)=0$. Hence we have $\Phi^{-1}(g Y)\left(p_{0}\right)=g\left(p_{0}^{\prime}\right) \Phi^{-1}(Y)\left(p_{0}\right)=$ $(g \circ \varphi)\left(p_{0}\right) \Phi^{-1}(Y)\left(p_{0}\right)$.

LEMMA 6.2. Let $\boldsymbol{R}^{1}$ be the one dimensional Euclidean space with the standard coordinate $x$. If $f: \boldsymbol{R}^{1} \rightarrow \boldsymbol{R}$ is a continuous function such that $g(x)=x \cdot f(x)$ is a $C^{r+1}$ function, then $f(x)$ is a $C^{r}$ function. Moreover if $g$ is a $C^{\infty}$ function, then $f$ is also a $C^{\infty}$ function.

Proof. It suffices to prove that $f$ is a $C^{1}$ function if $g$ is a $C^{2}$ function. Clearly $f$ is a $C^{2}$ function except the origin 0 . We take the Taylor expansion of $g(x)$ at 0 .

$$
g(x)=g(0)+g^{\prime}(0) \cdot x+\frac{1}{2} g^{\prime \prime}(\theta x) \cdot x^{2} \quad(0<\theta<1) .
$$

Since $g(x)=x \cdot f(x), g(0)=0$. So $x \cdot f(x)=g^{\prime}(0) \cdot x+\frac{1}{2} g^{\prime \prime}(\theta x) \cdot x^{2}$, and we have $f(x)=g^{\prime}(0)+\frac{1}{2} x g^{\prime \prime}(\theta x)$ for $x \neq 0$. Since $f(x)$ is continuous, $f(0)=$ $g^{\prime}(0)$. Hence we have 


$$
\lim _{x \rightarrow 0} \frac{f(x)-f(0)}{x}=\lim _{x \rightarrow 0} \frac{1}{2} g^{\prime \prime}(\theta x)=\frac{1}{2} g^{\prime \prime}(0) .
$$

Therefore $f(x)$ is differentiable at $x=0$ and $f^{\prime}(x)$ is continuous on $\boldsymbol{R}^{\mathbf{1}}$, that is, $f(x)$ is a $C^{1}$ function. By induction on $r, f(x)$ becomes a $C^{r}$ function.

COROLLARY 6.3. Let $\boldsymbol{R}^{m}$ be the Euclidean m-space with the standard coordinate $\left(x^{1}, \cdots, x^{m}\right)$. If $f: \boldsymbol{R}^{m} \rightarrow \boldsymbol{R}$ is a continuous function such that $g(x)=x^{1} \cdot f(x)$ is a $C^{r+1}$ function, then $f(x)$ is a $C^{r}$ function. Especially, if $g(x)$ is a $C^{\infty}$ function then $f(x)$ is also a $C^{\infty}$ function.

Proof. We regard $x^{2}, \cdots, x^{m}$ as smooth parameters of $g(x)$, and take the Taylor expansion of $g(x)$ at the origin $0 \in \boldsymbol{R}^{m}$ with respect to the first coordinate $x^{1}$. Then we can easily prove the differentiability of $f(x)$.

THEOREM 6.4. Let $\Phi: \Gamma_{0}\left(T_{M}\right) \rightarrow \Gamma_{0}\left(T_{M^{\prime}}\right)$ be a Lie algebra isomorphism. and $\varphi: M \rightarrow M^{\prime}$ be the induced homeomorphism. Then $\varphi$ becomes a $C^{\infty}$ diffeomorphism.

Proof. By Proposition 5.4, $\varphi: M-\left\{p_{0}\right\} \rightarrow M^{\prime}-\left\{p_{0}^{\prime}\right\}$ is a $C^{\infty}$ diffeomorphism. So it suffices to prove the differentiability of $\varphi$ at $p_{0} \in M$. Let $\left(U ; x^{1}, \cdots, x^{m}\right)$ be a local coordinate at $p_{0} \in M$. Then suitable extension of $x^{1} \cdot \partial / \partial x^{1}$ is contained in $\Gamma_{0}\left(T_{M}\right)$. We denote the extended vector field by $X$. Set $Y=\Phi(X)$, then $Y \in \Gamma_{0}\left(T_{M^{\prime}}\right)$. For any $g \in C^{\infty}\left(M^{\prime}\right)$ we set $Y_{1}=g Y$. Then $Y_{1} \in \Gamma_{0}\left(T_{M \prime}\right)$. Hence $X_{1}=\Phi^{-1}\left(Y_{1}\right)$ is contained in $\Gamma_{0}\left(T_{M}\right)$. By Lemma 6.1,

$$
X_{1}=\Phi^{-1}(g Y)=(g \circ \varphi) \Phi^{-1}(Y)=(g \circ \varphi) X .
$$

Hence, on the neighborhood $U, X_{1}=(g \circ \varphi) \cdot x^{1}\left(\partial / \partial x^{1}\right)$. Since $X_{1}$ is a $C^{\infty}$ vector field, $(g \circ \varphi) \cdot x^{1} \in C^{\infty}(U)$. By Proposition 4.7, $\varphi$ is continuous. Therefore the composition $g \circ \varphi$ is continuous and, by Corollary 6.3, $\varphi$ is $C^{\infty}$ differentiable at $p_{0} \in U \subset M$.

COROLlARY 6.5. Let $M$ and $M^{\prime}$ be compact manifolds without boundaries. If Lie algebras of $\mathscr{D}\left(M, p_{0}\right)$ and $\mathscr{D}\left(M^{\prime}, p_{0}^{\prime}\right)$ are isomorphic, then $\mathscr{D}\left(M, p_{0}\right) \cong \mathscr{D}\left(M^{\prime}, p_{0}^{\prime}\right)$ as I.L.H.-Lie groups.

Proof. Since Lie algebras of $\mathscr{D}\left(M, p_{0}\right)$ and $\mathscr{D}\left(M^{\prime}, p_{0}^{\prime}\right)$ are exactly $\Gamma_{0}\left(T_{M}\right)$ and $\Gamma_{0}\left(T_{M^{\prime}}\right)$, by Theorem 6.4,

$$
\mathscr{D}\left(M, p_{0}\right) \cong \mathscr{D}\left(M^{\prime}, p_{0}^{\prime}\right) \text {. }
$$


CoRollaRy 6.6. Let $\varphi: M \rightarrow M^{\prime}$ be the diffeomorphism induced by $\Phi$. Then we have $d \varphi=\Phi$.

Proof. Since $\Phi: \Gamma_{0}\left(T_{M}\right) \rightarrow \Gamma_{0}\left(T_{M^{\prime}}\right)$ is an isomorphism, for any $X \in \Gamma_{0}\left(T_{M}\right), \Phi(X) \in \Gamma_{0}\left(T_{M^{\prime}}\right)$. Since $\varphi: M \rightarrow M^{\prime}$ is a $C^{\infty}$ diffeomorphism, also we have $d \varphi(X) \in \Gamma_{0}\left(T_{M^{\prime}}\right)$. By Corollary $5.5 d \varphi(X)=\Phi(X)$ on $M^{\prime}-$ $\left\{p_{0}^{\prime}\right\}$ as $C^{\infty}$ vector fields. By continuity of the vector fields we have $d \varphi(X)\left(p_{0}^{\prime}\right)=\Phi(X)\left(p_{0}^{\prime}\right)$. Hence $d \varphi=\Phi$.

CoRollary 6.7. Let $N=\left\{p_{1}, \cdots, p_{s}\right\}$ and $N^{\prime}=\left\{p_{1}^{\prime}, \cdots, p_{t}^{\prime}\right\}$ be zero dimensional manifolds consisting of finite number of points. If $\Gamma_{N}\left(T_{M}\right)$ is isomorphic to $\Gamma_{N^{\prime}}\left(T_{M^{\prime}}\right)$, then $s=t$ and there exists a $C^{\infty}$ diffeomorphism $\varphi: M \rightarrow M^{\prime}$ such that $\varphi(N)=N^{\prime}$.

Proof. The proof is easy, and omitted.

(Case $\Gamma_{N}\left(T_{M}\right)$ with $\operatorname{dim} N \geqq 1$ )

LEMMA 6.8. Let $\Phi: \Gamma_{N}\left(T_{M}\right) \rightarrow \Gamma_{N^{\prime}}\left(T_{M^{\prime}}\right)$ be a Lie algebra isomorphism. We have then, for any $f \in C^{\infty}(M)$ and $X \in \Gamma_{N}\left(T_{M}\right), \Phi(f X)=\left(f \circ \varphi^{-1}\right) \Phi(X)$.

Proof. The proof is all the same as that of Lemma 5.2, and omitted.

THEOREM 6.9. Let $\Phi: \Gamma_{N}\left(T_{M}\right) \rightarrow \Gamma_{N^{\prime}}\left(T_{M^{\prime}}\right)$ be an isomorphism and $\varphi: M \rightarrow M^{\prime}$ be the induced homeomorphism. Then $\varphi$ is a $C^{\infty}$ diffeomorphism such that $\varphi(N)=N^{\prime}$.

Proof. Let $g$ be an arbitrary function in $C^{\infty}\left(M^{\prime}\right)$, and $q=\varphi(p)$ be an arbitrarily fixed point. Let $Y$ be any element of $\Gamma_{N^{\prime}}\left(T_{M^{\prime}}\right)$ such that $Y(q) \neq 0$. Actually we can take such $Y$, because of $\operatorname{dim} N^{\prime} \geqq 1$. We set $X=\Phi^{-1}(Y), Y_{1}=g Y$ and $X_{1}=\Phi^{-1}\left(Y_{1}\right)$.

(Case $p \notin N)$ By Lemma 5.1', $\left[Y, \Gamma_{N^{\prime}}\left(T_{M^{\prime}}\right)\right]+\mathscr{I}_{q}^{\prime}=\Gamma_{N^{\prime}}\left(T_{M^{\prime}}\right)$, where $\mathscr{I}_{q}^{\prime}$ is the maximal ideal corresponding to $q$. Since $\Phi$ is a Lie algebra isomorphism, by operating $\Phi^{-1}$ to the above equality we have $\left[X, \Gamma_{N}\left(T_{M}\right)\right]$ $+\mathscr{I}_{p}=\Gamma_{N}\left(T_{M}\right)$. Hence $X(p) \neq 0$.

(Case $p \in N) \quad$ By Lemma 5.1,$\quad\left[r_{*}^{\prime} Y, \Gamma\left(T_{N^{\prime}}\right)\right]+\overline{\mathscr{I}}_{q}^{\prime}=\Gamma\left(T_{N^{\prime}}\right) . \quad$ By operating the isomorphism $\Psi^{-1}: \Gamma\left(T_{N^{\prime}}\right) \rightarrow \Gamma\left(T_{N}\right)$, we have $\left(r_{*} X\right)(p) \neq 0$. Hence $X(p) \neq 0$.

So we may assume that $X=\partial / \partial x^{1}$ on a some neighborhood $U$ of $p$. On the other hand, $X_{1}=\Phi^{-1}\left(Y_{1}\right)=\Phi^{-1}(g Y)=(g \circ \varphi) \Phi^{-1}(Y)=(g \circ \varphi) X$. 
Hence $X_{1}=(g \circ \varphi)\left(\partial / \partial x^{1}\right)$ on $U$. This is an expression of the smooth vector field $X_{1}$ with respect to the local coordinate on $U$. Therefore $g \circ \varphi$ is contained in $C^{\infty}(M)$. So $\varphi$ is a diffeomorphism.

COROLlaRY 6.10. Let $\varphi: M \rightarrow M^{\prime}$ be the diffeomorphism induced by $\Phi$. Then we have $d \varphi=\Phi$.

Proof. The proof is same as that of Corollary 6.6.

\section{REFERENCES}

[1] L. Bishop and S. Goldberg, Tensor Analysis on Manifolds, The Macmillan Company, New York, 1968.

[2] D. G. Ebin and J. Marsden, Groups of diffeomorphisms and motion of an incompressible fluid. Ann. Math., 92 (1970) 102-163.

[ 3 ] Y. Matushima, Theory of Lie algebras (in Japanese), Kyoritu Shuppan.

[4] E. Nelson, TOPICS IN DYNAMICS I: FLOWS, Math. Notes, Princeton Univ. Press.

[ 5 ] H. Omori, On Lie algebras of vector fields, to appear.

[ 6 ] — On groups of diffeomorphisms on a compact manifold, Proc. Symp. Pure Math. A.M.S. 15 (1970) 168-183.

[ 7 ] — Groups of diffeomorphisms and their subgroups, to appear in Trans. A.M.S.

[8] - On infinite dimensional Lie transformation groups, to appear.

[9 ] L. E. Pursell and Shanks, The Lie algebra of a smooth manifold, Proc. Amer. Math. Soc., (1954) 468-472.

Department of Mathematics

Tokyo Metropolitan University

and

Department of Mathematics

Tokai University 